\title{
Protocolo de atención odontológica para el adulto mayor
}

\author{
Dental care protocol for the elderly \\ Carlos R. Davalos-Vargas ${ }^{a}$, Sara L. Monroy-Beltrán ${ }^{b}$, Jocelyn Muñoz-Benítez ${ }^{c}$, Valentín \\ Olvera-Flores ${ }^{d}$, Kenia E. Sánchez-Gómez ${ }^{e}$, Ana J. Monjarás Ávilaf
}

\begin{abstract}
:
The most vulnerable population in terms of oral diseases is considered to be the elderly or ancient, especially low-income patients or those who are systemically compromised. As patients age, their dental needs become increasingly specialized, making regular visits to the dentist even more essential; Furthermore, good hygiene is not as effective as before due to some bodily and motor limitations. That is why this work aims to highlight the primary needs for the care of older adults.
\end{abstract}

Keywords:

Elderly, treatment, oral diseases, prevention

\section{Resumen:}

Se considera que la población más vulnerable en cuanto a enfermedades orales son los adultos mayores o ancianos sobre todo los pacientes de bajos recursos o los que se están comprometidos sistémicamente. A medida que los pacientes envejecen, sus necesidades dentales se vuelven cada vez más especializadas, lo que hace que las consultas regulares al dentista sean aún más esenciales; además que la buena higiene no es tan efectiva como antes por algunas limitaciones corporales y motrices. Es por eso que este trabajo pretende resaltar las necesidades primordiales para la atención de los adultos de la tercera edad.

\section{Palabras Clave:}

Adulto mayor, tratamiento, enfermedades orales, prevención

\section{Introducción}

De acuerdo con la Comisión Económica para América Latina y el Caribe (CEPAL), México vive una fase de

\footnotetext{
${ }^{a}$ Estudiante Universidad Autónoma del Estado de Hidalgo, Instituto de Ciencias de la Salud. ORCID: https://orcid.org/0000-0002-51158153. e-mail: davalos.carlos32@gmail.com

${ }^{\mathrm{b}}$ Estudiante Universidad Autónoma del Estado de Hidalgo, Instituto de Ciencias de la Salud. ORCID: https://orcid.org/0000-0003-19078345. e-mail: monroyb.sara@gmail.com

${ }^{c}$ Estudiante Universidad Autónoma del Estado de Hidalgo, Instituto de Ciencias de la Salud. ORCID: https://orcid.org/0000-0002-71020493. e-mail: gynna.429@outlook.com

d Estudiante Universidad Autónoma del Estado de Hidalgo, Instituto de Ciencias de la Salud. ORCID: https://orcid.org/0000-0002-38704885. e-mail: olverava194@gmail.com

e Estudiante Universidad Autónoma del Estado de Hidalgo, Instituto de Ciencias de la Salud. ORCID: https://orcid.org/0000-0002-01218365. e-mail: kenia_27_04@hotmail.com

f Autor de Correspondencia, Universidad Autónoma del Estado de Hidalgo, https://orcid.org/0000-0002-5916-6510, Email: ana_monjaras@uaeh.edu.mx
} 
envejecimiento demográfico moderadamente avanzado; observando las cifras de la Encuesta Intercensal que indican que en 2015 había un total de 119443984 habitantes en el país, de los cuales, $10.4 \%$ corresponden a adultos mayores de 60 años o más.

Según la SIVEPAB las enfermedades orales que más predominan en los adultos mayores son caries, enfermedad periodontal, edentulismo total o parcial, cambios es la oclusión funcional y cáncer oral, como se observa en figura. ${ }^{1}$

En una población envejecida aparecerán con mayor prevalencia las denominadas patologías edad dependientes, son enfermedades cuya incidencia se incrementa con la edad y por ello la importancia de garantizar la existencia y acceso a servicios de atención médica adecuados que atiendan sus demandas ${ }^{1}$.

Por otra parte, la salud oral del paciente geriátrico se ve comprometida por hábitos de mala higiene, ya sea por alguna limitación motriz o simplemente no dar la importancia a la higiene oral, de igual manera no ser constante con la consulta periódica en el odontólogo; algunos de estos hábitos se muestran en la figura ${ }^{2}$.

Cuando atendemos a un paciente con alguna limitación motora, mental y visual, es indispensable indicar técnicas de aseo sencillas pero efectivas e implementar el uso de cepillos e hilo dental modificados a las necesidades de cada individuo porque, de lo contrario, las técnicas no tendrán los resultados esperados y aumentaría la frustración por parte del paciente.

Los adultos mayores presentan desventajas como la mayor exposición a enfermedades, sobre todo crónico degenerativas e incapacitantes, muestran también algunos factores sociodemográficos por los que se ven afectados como las condiciones de dependencia económica y pobreza, pérdida de las capacidades físicas y mentales y disminución en su grado de autonomía y adaptabilidad como se muestra en la figura ${ }^{3}$.

El ser humano pasa por diferentes etapas durante su desarrollo, en los cuales sufre muchos cambios tanto a nivel físico como psicológico. El envejecimiento es un proceso de cambios anatómicos y fisiológicos dependientes del tiempo que reducen la capacidad funcional y fisiológica del cuerpo. ${ }^{2}$

Conforme avanza la edad, el cuerpo sufre muchos cambios morfológicos a nivel de sus tejidos y sistemas; en la mayoría de los casos, la salud del individuo se ve comprometida debido a problemas dentales, nutricionales, mentales y por patologías sistémicas (cáncer, hipertensión, diabetes mellitus, insuficiencias cardiacas, entre otros) ${ }^{3}$.

\section{Caries dental \\ - La severidad de caries dental es mayor en los adultos, el número de dientes afectados para este grupo de edad se encuentra en el rango de 16.6 a 17.9 dientes en los últimos diez años}

\section{Enfermedad Periodontal}

- Se observa que las bolsas periodontales mas profundas afecta al grupo de edad de 65 a 74 años ocupando el $31.5 \%$ a $39.0 \%$ de toda la población con una prevalencia en mujeres

\section{Edentulismo}

- El porcentaje de adultos con ausencia total de dientes naturales, se ubicó en el rango de $1.02 \%$ a $2.65 \%$ segun la OMS. Los resultados del SIVEPAB a lo largo de estos 10 años han demostrado que el edentulismo es más frecuente en el sexo femenino que en el masculino

\section{Oclusión Funcional}

- Para el grupo de edad de 65 a 74 años la proporción de pacientes con oclusión funcional disminuye a un rango de 63.9 a $69.2 \%$ indicando una deficiencia masticatoria

\section{Cáncer Oral}

- El cáncer de la cavidad oral es el octavo cáncer más frecuente en los hombres que en mujeres ocupando el $25 \%$ de la población

Figura 1. Epidemiología de enfermedades orales del adulto mayor en México según la SIVEPA.

Algunos cambios que se presentan en los tejidos orales de pacientes geriátricos son la producción continua de dentina ocasionando que la cámara pulpar reduzca su tamaño, así como los canales radiculares, vasos sanguíneos y fibras periodontales. Con la edad aparecen pigmentaciones en mucosa oral y piel, que se deben en la mayoría de los casos a una melanosis fisiológica o racial y pueden aparecer en cualquier región de la boca como carrillos, labios, lengua y encía ${ }^{4}$.

El proceso de envejecimiento produce en la cavidad oral una serie de cambios graduales, irreversibles y acumulativos, lo que origina en ella una mayor vulnerabilidad a los agentes traumáticos e infecciosos. Los cambios que se presentan en los tejidos son:

\section{- Periodonto}

A nivel celular, se presentan diferencias en el espesor de las capas celulares, entre la capa basal y la córnea. Con el envejecimiento, el periodonto se engrosa y se retrae exponiendo mayor superficie dental al medio bucal ${ }^{5}$.

El exceso y desequilibrio de las fuerzas oclusales, producto de la pérdida de piezas dentales, provoca que el cemento se engrose a nivel apical en las piezas remanentes en forma benigna o como neoplasia del cemento dental $^{6,7}$. 


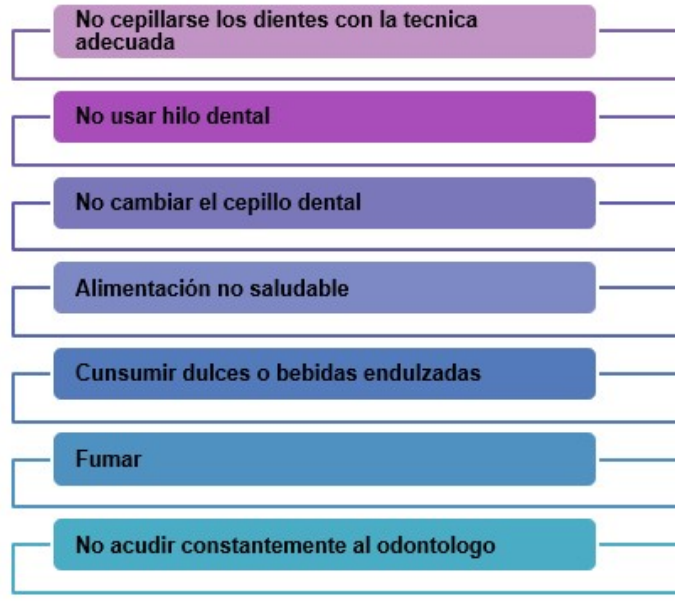

Figura 2. Hábitos de la mala higiene y la no prevención de patologías bucodentales.

\section{- Mucosa Oral}

La mucosa oral, al igual que la piel, sufre un decrecimiento en el grosor debido a la disminución en la microvasculatura. La estructuración celular varía, debilitando la mucosa y facilitando las infecciones, desgarros, lesiones precancerosas y cancerosas de cavidad oral. La mucosa oral puede presentar otro signo no característico del envejecimiento, como la sequedad, producto de la xerostomía. Esta se produce por a la presencia de deshidratación, respiración bucal, enfermedades de fondo o tratamientos farmacológicos ${ }^{8}$.

Hay que recordar que con la edad aparecen pigmentaciones en mucosa oral y piel, que se deben en la mayoría de los casos a una melanosis fisiológica o racial y pueden aparecer en cualquier región de la boca como carrillos, labios, lengua (dorso y lateral) y encía. No obstante, es importante hacer un diagnóstico diferencial para descartar lesiones y pigmentaciones asociadas al tabaquismo (melanosis del fumador), traumas crónicos, cáncer y las secundarias a medicamentos.

\section{- Labios}

La pérdida de piezas dentales, así como de elasticidad muscular ocasiona que el músculo orbicular de los labios se quede sin soporte y por consecuencia tenga una apariencia de flacidez (hipotonicidad muscular).

Esto ocasiona que la piel de los labios se arrugue hacia adentro. De esta manera, la barbilla se ve pronunciada, a esto se le conoce como pseudo prognatismo ${ }^{8}$.

\section{- Glándulas Salivales}

Con el envejecimiento, se produce una atrofia del tejido acinar y una proliferación de productos ductales. Es por eso que las glándulas salivales mayores como las glándulas salivales menores atraviesan por un proceso de cambios degenerativos conforme el cuerpo envejece. Los cambios glandulares se muestran en la Figura 4.

Estos cambios también son ocasionados por medicamentos o radiación en la zona de cabeza y cuello. Se ha reportado que algunas enfermedades crónicas y reacciones atróficas de la mucosa palatina están asociadas con reducción del flujo salival palatino, estrés, depresión, abuso de nicotina y alcohol, deficiencia de estrógeno. Además, efectos adversos de drogas tales como los digitálicos, tranquilizantes y antidepresivos tricíclicos contribuyen decididamente a una hiposecreción de las glándulas. ${ }^{9}$

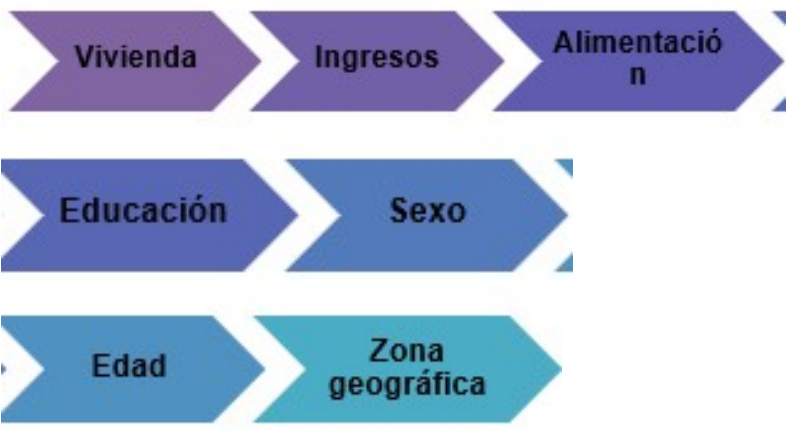

Figura 3. Factores sociodemográficos que afectan la salud oral del adulto mayor.

- Saliva

La saliva es importante para el buen funcionamiento de todas las estructuras de la cavidad bucal y para mantener la salud oral. También facilita los fenómenos sensoriales y motores, protege el tracto digestivo y orofaríngeo. En el envejecimiento, la producción de saliva no se ve comprometida. Se cree que esto se debe a la capacidad de reserva funcional de las glándulas lo que permite continuar con su producción a través de la vida del adulto. La calidad y cantidad de la saliva puede verse afectada debido a la presencia del Síndrome de Sjögren, radiación de cabeza y cuello, quimioterapia citotóxica, influencia de medicamentos, patologías, entre otros ${ }^{9,10}$.

\section{- Lengua}

La ausencia de piezas dentales inferiores origina un ensanchamiento lingual producto del sobre desarrollo de la musculatura intrínseca. En ocasiones, en la lengua, se puede ver un aumento de varices en la superficie ventral y tener una apariencia lisa y dolorosa. El número de papilas gustativas no cambia con la edad ${ }^{11}$.

\section{- Dientes}

La producción continua de dentina provoca que la cámara pulpar disminuya su tamaño, así como los canales radiculares, vasos sanguíneos y fibras periodontales. Esto demuestra que la respuesta pulpar ante factores 
agresores se vea disminuida y que los tratamientos endodónticos se restringen con la edad ${ }^{12}$.

Por otro lado, el oscurecimiento y color amarillo de los dientes es resultado de cambios en el grosor y composición de la dentina. La dentina se caracteriza por contener menor cantidad de túbulos y mayor densidad entre ellos (dentina esclerótica).

El esmalte toma una contextura rugosa que contribuye más fácilmente a la adhesión de pigmentos produciendo manchas dentales.

Además, se pueden observar signos de desgaste en las piezas dentales en forma de abrasión, abfracción, erosión y atrición marcada producto del deterioro fisiológico característico de la masticación, desgastes mecánicos o químicos y patologías como el bruxismo ${ }^{13}$.

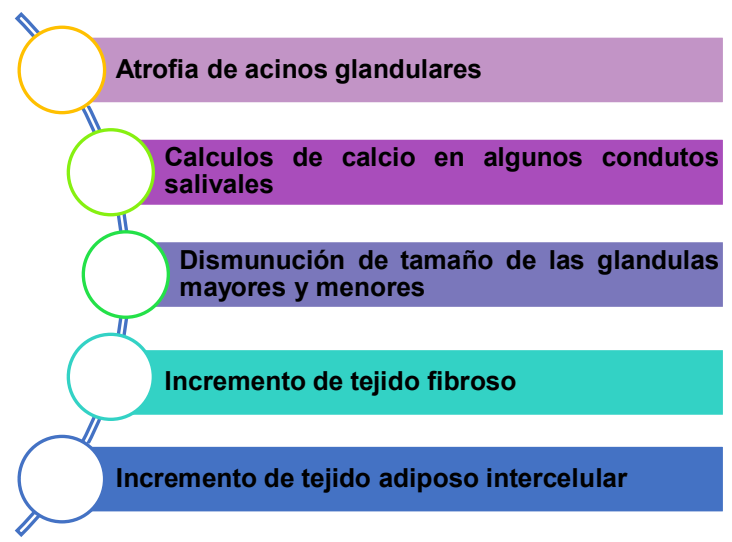

Figura 4. Cambios degenerativos de las glándulas salivales.

\section{Salud oral en el adulto mayor}

Una regular cobertura preventiva es igual de importante para el grupo de la tercera edad que para las otras etapas de la vida. En la mayoría de los adultos mayores faltan, por lo general, piezas dentales y el número de sus restauraciones es elevado. Esto por sí solo representa una preocupante necesidad de estrategias preventivas en los planes de tratamiento de estos pacientes ${ }^{14}$.

Sin embargo, también existe un creciente aumento de ancianos de bajos recursos, limitando sus posibilidades de invertir en costosos tratamientos dentales. Igualmente encontramos un preocupante número de personas de edad erróneamente persuadidas de la idea de que la pérdida de los dientes y los cambios físicos y nutricionales que acarrea este hecho es parte inevitable del envejecimiento.

En muchos casos se torna difícil la continuidad de una terapia restaurativa (transporte hacia la consulta dental, efectos secundarios de anestésicos locales, peligro de complicación ante extracciones, etc.), lo que aumenta la importancia de la conservación de la salud bucal a través de apropiadas estrategias de prevención ${ }^{15}$.

El tratamiento se torna en especial difícil cuando se llega a estados en los que estos casos necesitan la asistencia de terceros, cuando la higiene oral no puede seguir siendo efectuada de manera eficiente en forma autónoma, en pacientes incapacitados para valerse por sí mismos (parálisis, Parkinson, Alzheimer, etc.) ${ }^{16}$.

En general se puede decir que desde el punto de vista psicológico el grupo de la tercera edad es muy heterogéneo. Muchos ancianos mantienen una vida activa en la que adoptan nuevas actividades, las que a menudo desarrollan con gran entusiasmo. Desean no ser tratados como "viejos".

\section{Manejo odontológico del paciente geriátrico}

Todos los órganos y sistemas del cuerpo humano envejecen. No todos al mismo ritmo, pero ninguno está exento de las modificaciones que conlleva el paso del tiempo. El sistema estomatognático, no es la excepción. Modificaciones extraorales e intraorales hacen que se tengan unas condiciones, necesidades $y$ susceptibilidades diferentes en esta etapa de la vida ${ }^{17,18}$.

Las modificaciones que se presentan en el sistema estomatognático son variadas y para ello se debe seguir una serie de cuidados en la atención del paciente geriátrico. En el siguiente diagrama se mostrará el protocolo de cuidados odontológicos en el paciente geriátrico (Figura 5).

Ante este protocolo de atención oral al paciente geriátrico es indispensable hacer una valoración y formar parte de equipos multidisciplinarios que sume la participación de profesionales orientados a comprender el manejo de pacientes con situaciones especiales, como la demencia y diferentes tipos de discapacidad, para adaptar los enfoques que tienen los odontólogos a las necesidades propias de cada individuo y sus condiciones de vida, como son el médico internista, neurólogo, ortopedista, el psicólogo, la enfermera, el implantológo, el gnatologo, prótesista, periodoncista, por mencionar algunos, de forma que se tenga la capacidad de adaptar los conocimientos de los profesionales a las necesidades individuales, con las especificidades de cada caso y que se ajusten a la realidad en la que viven las personas mayores $^{19}$.

La prevención se considera como el punto más importante que se debe llevar desde edades tempranas para tener una mejor calidad de vida y así conservar un mayor número de dientes en la vejez.

Otro punto relevante es el manejo adecuado en diagnóstico y terapéutica que garantice las condiciones de salud oral durante el curso del envejecimiento. 
Comprender las limitaciones propias de los cambios que sufre el sistema estomatognático con la vejez, para abordarlos de la manera más adecuada, en la planeación de la atención de los pacientes geriátricos ${ }^{20}$.

Existe un estudio que desarrollo la odontología para pacientes geriátricos, en la cual se diseñan los tratamientos desde cinco aspectos importantes que valoran la condición del anciano, propuestos por la Escuela Norteamericana, que por sus siglas se resumen en $\mathrm{OSCAR}^{20}$, que podemos observar en la Figura 6.

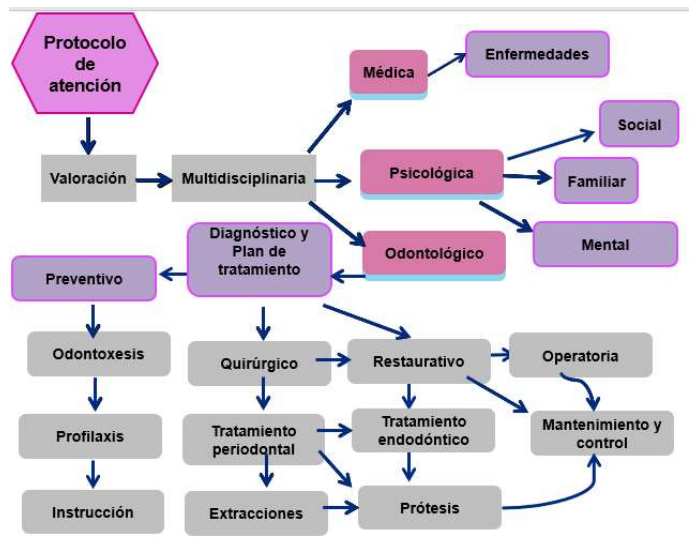

Figura 5. Protocolo de atención odontológica del paciente adulto.

Estos aspectos se valoran desde que se establece el primer contacto con el paciente, y se obtienen no sólo desde la elaboración de la historia clínica, sino también desde la observación de comportamientos y conductas específicas del paciente y sus acompañantes.

Pensar en el orden de prioridades se ajusta a los períodos clásicos de tratamiento odontológico y a la correcta interpretación del estado del paciente, lectura que puede hacerse desde que comienza la anamnesis, complementando con el examen clínico y finalizando con la interacción y capacidad de comunicación que se establece con el paciente y su cuidador ${ }^{21}$.

\section{Medidas preventivas}

El envejecimiento de la cavidad bucal, es un proceso tremendamente influenciable por factores diversos como el estilo de vida de la persona, el tipo de alimentación, el nivel educativo, los factores socioeconómicos, soporte social, etc. Estos hechos observados se encuentran asociados íntimamente con alteraciones en el estado de la salud oral en el adulto mayor. ${ }^{22}$

Frecuentemente nos encontramos con que el único tratamiento que creen necesitar de parte del profesional es una mera intervención de reparación para eliminar la molestia, es por eso que se recomienda enfocar el tratamiento de acuerdo a la prioridad que el paciente presente, como se explica en la Figura 7.

La anamnesis debiera contemplar un meticuloso análisis médico-dental, que comprenda una actualización permanente de las enfermedades y medicamentos. También puede ocurrir que el paciente no recuerde el nombre de los medicamentos que ingiere, o que no sepa la causa por la cual le fueron prescritos. En caso de duda, será necesario pedir que lleve en mano sus medicamentos o en su defecto la receta, en su próxima sesión, de lo contrario será necesario llamar al médico tratante ${ }^{23}$.

En general, es esencial que, al inicio del tratamiento, se determine qué posición puede asumir el paciente en el sillón dental. El tiempo del tratamiento debiera compatibilizarse con las condiciones propias del paciente, como ser sus limitaciones físicas en la apertura de la boca que puede ser limitada o dificultosa ${ }^{24}$.

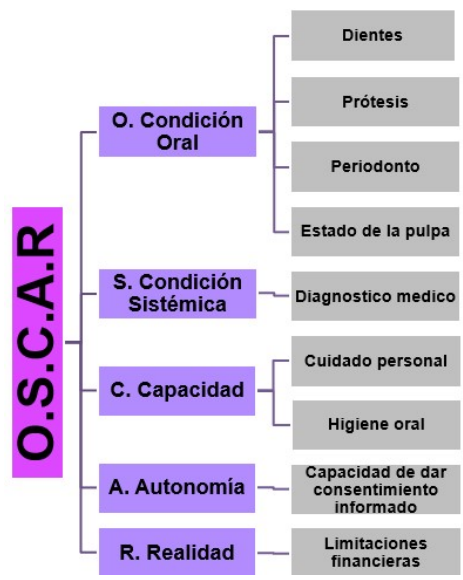

Figura 6. Aspectos que valoran la condición del paciente geriátrico.

\section{Manejo de la salud bucal en paciente geriátrico con demencia}

La demencia se clasifica como: "Una enfermedad de carácter neurodegenerativo, que involucra la alteración de múltiples funciones corticales superiores, acompañadas por un deterioro del control emocional, del comportamiento social o de la motivación, que se han agrupado como síntomas en tres dimensiones: cognitiva, funcional y del comportamiento ${ }^{25}$.

Las enfermedades orales más comunes encontradas en pacientes ancianos y más aún con demencia o algún tipo de discapacidad, se asocian a la presencia de placa bacteriana: caries dental y enfermedad periodontal, debido principalmente a la dificultad de realizar la higiene oral, a los cambios en la dieta y a la 
hiposalivación relacionada con el consumo de medicamentos ${ }^{26}$.

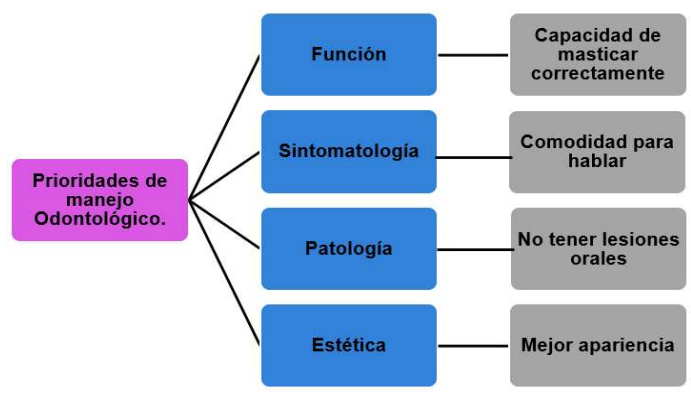

Figura 7. Factores dominantes para enfocar las prioridades del manejo de las necesidades odontológicas en pacientes geriátricos.

Es común en los pacientes con demencia usuarios de prótesis bucales, encontrar las prótesis en condiciones regulares comprometiendo la salud de los tejidos circundantes. A esto, se le suman factores externos que pueden complejizar la prevalencia de enfermedades orales como: tipo de vivienda, dificultad de acceso a los servicios de salud, dependencia económica y dificultad de comunicación que irá en aumento en proporción directa con el progreso de la enfermedad ${ }^{27}$.

Dado lo anterior una vez realizado el diagnóstico de demencia, es importante entrar en un plan a largo plazo de lo que será su atención odontológica; entendiendo que, con el tiempo y la evolución de la enfermedad, cada vez será más complejo realizar una atención directa del paciente, a este le será más difícil realizar la higiene oral de forma autónoma y por ende será más complejo mantener una buena salud oral. Por lo tanto, el pronóstico de salud oral mejorará, en la medida que al paciente se involucre desde las etapas tempranas de la demencia en el tratamiento y el manejo odontológico; debido a que es más receptivo al tratamiento y puede tener un papel más activo en la toma de decisiones ${ }^{28}$.

Si todavía hay colaboración y comprensión del paciente para llevar a cabo la atención dentro del consultorio odontológico, los objetivos del plan de tratamiento deben ser enfocados en dos aspectos: Promoción y prevención, con énfasis no solo al paciente, sino también en involucrar a sus familiares y cuidadores, identificando los factores de riesgo individuales ${ }^{29}$.

En esta etapa de promoción y prevención se deben eliminar factores locales que aumenten el riesgo:

- Ajuste de restauraciones y rehabilitaciones protésicas.

- Uso de cepillos eléctricos, o con una adaptación especial en el mango que son de gran ayuda.
- Prescripción de pastas dentales con fluoruro en altas concentraciones.

- Para la higiene interproximal pueden ser más eficaces el uso de cepillos, que el de la seda dental.

En la etapa de prevención y promoción también se pueden realizar procedimientos restauradores simples como por ejemplo algún cambio de un tratamiento operatorio o ajuste de prótesis pero sin ser un tratamiento de rehabilitación complejo, pensando siempre que el diseño de todo tipo de restauraciones deberá ser de fácil limpieza, debido a la forma como este aspecto se dificultará a medida que progrese la demencia, de forma que si el paciente pierde su posibilidad de realizar la higiene para el futuro cuidador sea más fácil hacerlo ${ }^{30}$.

\section{Conclusiones}

Los adultos mayores constituyen un grupo vulnerable por ser susceptible a trastornos bucodentales que afectan su estado de nutrición, la percepción que tienen de sí mismos y sus relaciones sociales. Estas necesidades tienen que ser evaluadas antes de que impacten en su calidad de vida, sin embargo, debido a la creciente demanda de atención de este sector de población en todos los países, se constituye en un importante problema de salud pública que debe ser abordado.

Cuando el paciente geriátrico presente alguna patología de fondo, el odontólogo tratante debe investigar si el paciente está bajo tratamiento médico y si el cuadro se encuentra estabilizado, antes de realizar cualquier procedimiento por más sencillo que sea. De lo contrario, el paciente debe ser remitido a su médico para una valoración, aunque esto implique postergar el tratamiento dental.

\section{Referencias}

[1] Gualotuña Tipán S. Factores de riesgo relacionados con la aparicion de lesiones bucales en paciente geriátricos en el Hospital de atencion Integral del adulto mayor, Quito 2015-2016. 2015;

[2] Blanco Mederos FM. Consideraciones actuales sobre la Estomatología Geriátrica y su importancia para una longevidad saludable Current considerations on geriatric Dentistry and its importance for a healthy longevity. Rev Med Electron [Internet]. 2017;39(1):70-83. Available from: http://scielo.sld.cu/pdf/rme/v39n1/rme090117.pdf

[3] Mejía-Pérez PN, Arbeláez-Lelión D, Múnera MC. Una mirada al manejo odontológico del paciente geriátrico con demencia. CES Odontol. 2017;30(1):51-67.

[4] Chávez-Reátegui BDC, Manrique-Chávez JE, Manrique-Guzmán JA. Odontogeriatría y gerodontología: el envejecimiento y las características bucales del paciente adulto mayor: Revisión de literatura. Rev Estomatológica Hered. 2014;24(3):199. 
[5] Paez González Y, Tamayo Ortiz B, Batista Bonillo A, García Rodríguez Y, Guerrero Ricardo I. Factores de riesgo de periodontopatías en pacientes adultos. Correo Científico Médico. 2015;19(2):269-81.

[6] Carvajal P. Enfermedades periodontales como un problema de salud pública: el desafío del nivel primario de atención en salud. Rev Clínica Periodoncia, Implantol y Rehabil Oral [Internet]. 2016;9(2):177-83. Available from: http://dx.doi.org/10.1016/j.piro.2016.07.001

[7] Rev CO, Camag AM, Hern B, Mar S, Hidalgo SH, Garc L, et al. Factores de riesgo de bruxismo en pacientes adultos. Arch Médico Camagüey. 2017;21(3):311-20.

[8] Cabo, R., Grau. B. I. y Lorenzo, M. (2016). Apuntes sobre el envejecimiento del sistema estomatognático. Revisión de la literatura. Rev. Medisur. 14 (3)

[9] León S, Castro E, Arriagada K, Giacaman RA. Flujo salival y caries radicular en adultos mayores autovalentes. Rev Clínica Periodoncia, Implantol y Rehabil Oral [Internet]. 2016;9(3):253-8. Available from: http://dx.doi.org/10.1016/j.piro.2016.09.003

[10] Díaz-Ramos JA, Mondragón-Cervantes MI, Jiménez-Acosta Y del C, Fraga-Ávila C, Tostado-Flores LM, Presa-Ramírez JC, et al Prevalencia de la xerostomía y su asociación con síndromes geriátricos en pacientes ancianos que asisten a un centro de atención de primer nivel TT. Rev odontol mex [Internet]. 2018;22(4):214-20. Available from:

http://www.scielo.org.mx/scielo.php?script=sci_arttext\&pid=S1870199X2018000400214

[11] Díaz, S. L., Jiménez, I. M., Paez, D. y Cols. (2018). Enfermedades bucales y factores de riesgo en la población geriátrica perteneciente a un consultorio médico. Rev. Medisan 22 (5).

[12] Ministerio de Salud. Salud Oral Integral para adultos de 60 años: Prevención y tratamiento de caries radiculares. Guía Clínica AUGE Salud Oral Integr para adultos 60 años Prevención y Trat caries radiculares [Internet]. 2015;39. Available from: http://www.bibliotecaminsal.cl/wp/wpcontent/uploads/2016/04/Salud-Oral-Integral-para-adultos-de-60años-radicular.pdf

[13] Azañedo D, Díaz-Seijas D, Hernández Vásquez AA. Oral health of the elderly: Access, challenges and opportunities for Peruvian public health. Rev Peru Med Exp Salud Publica. 2016;33(2):373-5.

[14] Díaz, S. L., Jiménez, I. M., Paez, D. y Cols. (2018). Enfermedades bucales y factores de riesgo en la población geriátrica perteneciente a un consultorio médico. Rev. Medisan 22 (5).

[15] Razak PA, Richard KMJ, Thankachan RP, Hafiz KAA, Kumar KN, Sameer KM. Geriatric oral health: a review article. J Int oral Heal $\mathrm{JIOH}$ [Internet]. 2014;6(6):110-6. Available from: http://www.ncbi.nlm.nih.gov/pubmed/25628498\%0Ahttp://www.pub medcentral.nih.gov/articlerender.fcgi?artid=PMC4295446

[16] Dho MS. Factores asociados a la utilización de servicios de salud odontológicos. Cienc e Saude Coletiva. 2018;23(2):509-18.

[17] Córdova Sotomayor DA, Santa Maria Carlos FB. Niveles de ansiedad en pacientes adultos de una clínica odontológica en una universidad peruana. Rev Estomatológica Hered. 2018;28(2):89

[18] Fernández E, Padilla P, Monardes H, Díaz C. Actitud hacia el adulto mayor en estudiantes del pregrado de la Facultad de odontología
Universidad San Sebastián, Santiago. Rev Estomatológica Hered. 2017;27(1):21

[19] Rebolledo Cobos M, de la Cruz Villa A, Hernández Miranda K, Brigitte Núñez B. A RTÍCULO O RIGINAL Lesiones bucales en pacientes adultos mayores con hipertensión arterial de una institución geriátrica de Barranquilla. Oral lesions in elderly patients with hypertension from a geriatric institution in Barranquilla. Hernández, Núñez Rev CSV. 2018;10(1):4-14

[20] Valenzuela MJ. Buscando un mejoramiento en la salud oral de los adultos mayores: ¿Es necesaria una reforma al GES-60 años? Rev Chil Salud Pública. 2015;19(2):181.

[21] Pardhan MS, Sonarkar SS, Shenoi PR, Uttarwar V, Mokhade V. Geriatric Dentistry-an Overview. Int J Oral Heal Dent. 2016;2(1):26.

[22] Grover S. Geriatric Dentistry : From Entry to Exit and Beyond. 2018;(September).

[23] Haya Fernández MC, Blasco Garrido I, Cabo Pastor MB. La atención odontológica del paciente geriátrico con deterioro cognitivo. Av Odontoestomatol. 2015;

[24] Original A, Gavilanes-bayas NA. pacientes edéntulos totales Oral mucosal lesions associated with the use of dental prostheses in total edentulous patients Lesões da mucosa bucal associadas ao uso de prótese dentária em pacientes totalmente desdentados. Ciencias Médicas. 2019;5:603-23.

[25] Hempel FM, Krois J, Paris S, Beuer F, Kuhlmey A, Schwendicke F. Prosthetic treatment patterns in the very old : an insurance database analysis from Northeast Germany. 2020;

[26] Alzate-Urrea S, Agudelo-Suárez AA, López-Vergel F, López-Orozco C, Espinosa-Herrera É, Posada-López A, et al. Calidad de vida y salud bucal: Perspectiva de adultos mayores atendidos en la red hospitalaria pública de Medellín, Colombia. Rev Gerenc y Polit Salud. 2015;14(29):83-96.

[27] Salas AC. Determinantes sociales y condiciones de salud bucal de los adultos mayores Social determinants and oral health conditions in the elderly. 2019;56(5):174-86.

[28] Hoeksema AR, Peters LL, Raghoebar GM, Meijer HJA, Vissink A, Visser A. Oral health status and need for oral care of care-dependent indwelling elderly: from admission to death. Clin Oral Investig. 2017;21(7):2189-96.

[29] Schwendicke F, Nitschke I, Stark H, Micheelis W, Jordan RA. Epidemiological trends, predictive factors, and projection of tooth loss in Germany 1997-2030: part II. Edentulism in seniors. Clin Oral Investig. 2020;

[30] Yi Mohammadi JJ, Franks K, Hines S. Effectiveness of professional oral health care intervention on the oral health of residents with dementia in residential aged care facilities: a systematic review protocol. JBI database Syst Rev Implement reports. 2015;13(10):110 22.Dilla T, Valladares A, Lizán L, Sacristán JA. Adherencia y persistencia terapéutica: causas, consecuencias y estrategias de mejora. Aten. Primaria 2009; 41(6): 342-48. 